sensitive to the value of $j$ and thus provides a good way of determining it. They obtained the vector analysing power from the left-right asymmetry of the protons emitted from a reaction initiated by a polarised beam of particles. The $j=\frac{1}{2}^{-}$reactions ${ }^{32} \mathrm{Cr}(\mathrm{d}, \mathrm{p})^{53} \mathrm{Cr}$ $(Q=5.17 \mathrm{MeV})$ and ${ }^{54} \mathrm{Fe}(\mathrm{d}, \mathrm{p}){ }^{55} \mathrm{Fe}$ $(Q=6.60 \mathrm{MeV})$ at $10 \mathrm{MeV}$ had vector analysing powers quite different from the $j=3 / 2^{-}$reactions ${ }^{52} \mathrm{Cr}(\mathrm{d}, \mathrm{p})^{53} \mathrm{Cr}$ $(Q=5.73 \mathrm{MeV})$ and ${ }^{54} \mathrm{Fe}(\mathrm{d}, \mathrm{p}){ }^{55} \mathrm{Fe}(Q=$ $7.01 \mathrm{MeV})$ at $10 \mathrm{MeV}$. In the mixed- $j$ reaction ${ }^{53} \mathrm{Cr}(\mathrm{d}, \mathrm{p}){ }^{54} \mathrm{Cr} \quad(Q=6.71 \mathrm{MeV})$ at $10 \mathrm{MeV}$, both these values of $j$ were possible, and the vector analysing power closely followed a curve obtained by adding together in definite proportions the vector analysing powers found in the other two reactions. This proportion gave the relative contributions of the $j=\frac{1}{2}$ and $j=3 / 2$ components to the reaction. The technique of finding the mixing parameter in mixed- $j$ transitions has the special advantage of being independent of detailed theoretical calculations, but is somewhat laborious to apply in practice.

A new method of finding the $j$ value has recently been proposed by Dohan and Summers-Gill (Nuclear Phys., A241, 61; 1975), and applied to the ${ }^{69} \mathrm{Gd}(\mathrm{d}, \mathrm{p}){ }^{70} \mathrm{Gd}$ and ${ }^{71} \mathrm{Gd}(\mathrm{d}, \mathrm{t})^{70} \mathrm{Gd}$ reactions to the same state of ${ }^{70} \mathrm{Gd}$. It depends on the fact that, on the simple shell model, the $2 \mathrm{p}_{1 / 2}$ neutron state is empty in ${ }^{69} \mathrm{Gd}$, half-full (containing one neutron) in ${ }^{70} \mathrm{Gd}$ and full in ${ }^{71} \mathrm{Gd}$. Thus the $(\mathrm{d}, \mathrm{p})$ and $(\mathrm{d}, \mathrm{t})$ reactions on ${ }^{69} \mathrm{Gd}$ and ${ }^{71} \mathrm{Gd}$ respectively go equally well, and thus have very similar spectroscopic factors. The $2 \mathrm{p}_{3 / 2}$ state, on the other hand, is full for all these nuclei, so the $(\mathrm{d}, \mathrm{p})$ reaction on ${ }^{69} \mathrm{Gd}$ is forbidden, as there is no room for another neutron, while the $(d, t)$ reaction on ${ }^{71} \mathrm{Gd}$ takes place easily as there are plenty of $2 \mathrm{p}_{3 / 2}$ neutrons to be removed. This picture is expected to be somewhat blurred as the simple shell model is not followed exactly, but nevertheless we expect to find very similar amplitudes for $2 \mathrm{p}_{1 / 2}$ transfer and very different amplitudes for $2 \mathrm{p}_{3,2}$ transfer in the two reactions.

In practice these expectations are remarkably well fulfilled. The transitions to the lower states up to about $1 \mathrm{MeV}$ have almost the same amplitudes for the two types of reaction while those above $1 \mathrm{MeV}$ have very small amplitudes for the $(d, p)$ reaction and large amplitudes for the $(d, t)$ reaction. Thus we can with some confidence assign the first group to $2 \mathrm{p}_{1 / 2}$ transfer and the second group to $2 p_{3 / 2}$ transfer. This is a very simple and direct way of determining the $j$ value of the transferred particle, and is applicable to reactions where the subshell corresponding to one $j$ value is filling more rapidly than the other. It will be interesting to see how far it can be extended.

In heavy ion reactions the situation is more complicated since the transferred particle can have an angular momentum greater than zero in the projectile. Thus the neutron in the deuteron has $l=0$, whereas the proton in ${ }^{7} \mathrm{Li}$ that is transferred by the $\left({ }^{7} \mathrm{Li},{ }^{6} \mathrm{He}\right.$ ) reaction has $l=1, j=3 / 2$ as it comes from an $\mathrm{p}_{3 / 2}$ orbit in ${ }^{7} \mathrm{Li}$ (Nature, 253, 501; 1975).

\section{Sticky actin}

from Dennis Bray

MuscLe biochemistry is not usually the place to look for the bizarre; but what else could one call an association between muscle actin and an enzyme that digests DNA? The story is a curious one. As long ago as 1943, it was fcund that pigeon thymus contains a protein that inhibits deoxyribonuclease I-the abundant endonuclease of pancreas. This activity was found in other tissues but little else was learnt about its biochemistry until the late 60 s when Lindberg - at the Karolinska Institute, Stockholm-began a careful study. He found that the inhibitor had a molecular weight close to 42,000 ; that it could be purified and even crystallised by conventional procedures; and that it formed a tight $1: 1$ complex with the nuclease. Among its notable characteristics, at least in retrospect, was its abundance in tissue $(5-10 \%$ of the protein in a soluble extract), and its tendency to form aggregates of high molecular weight.

Then last year, at cold spring Harbor, New York, Lazarides and others interested in cell motility heard at first-hand of the DNase inhibitor. They also had a protein with molecular weight close to 42,000 which was strangely abundant in cells: only they called it actin. The amino acid compositions of the two proteins were encouragingly similar and the unlikely possibility was put to the test. Sure enough, the fingerprints of the two are the same, they cross react immunolog.cally, and analytical-grade muscle actin sticks so tightly to DNase I that denaturing agents are needed to part them (Lazarides and Lindberg, Proc. natn. Acad. Sci. U.S.A., 71, 4742; 1974).

The interaction is unquestionably real but why does it occur? It is unlikely to be accidental because the binding is so tight. It may have some purpose, but it links two cellular processes that are normally considered to be independent. The puzzle is compounded by indications that actin forms other unexpected associations. Before the work mentioned above, Laki and Muszbek (Biochim. biophys. Acta.,
371, 519; 1974), had shown that actin in its filamentous form binds strongly to fibrin--the major structural protein of blood clots. And a recent paper examines the long known association between glycolytic enzymes, such as aldolase and triose phosphate dehydrogenase, and filamentous actin (Clark and Masters, Biochim. biophys. Acta., 381, 37; 1975). Still only in abstract form are claims that an interaction exists between actin and spectrin, the major protein of red blood cell ghosts (Tilney, J. Cell Biol., 63, 349a; 1974); and another report that actin might be identical to the $\gamma$-subunit of dogfish muscle phosphorylase (Fischer et al., Hoppe-Seylers Z. Sïr Physiol. Chem., 356, 381; 1975)

If we consider the proteins that associate with thin filaments in muscle -troponin, tropomyosin, myosin and actinin-then we have ten or so that all bind to actin. The list might well be longer, for any club that includes both deoxyribonuclease and myosin must surely have a wide membership. It begins to look as though we are in the presence of a Phenomenon; but what it might mean is hard to say. Fither actin is generally sticky and able to adhere to proteins it does not normally encounter, or many proteins have cvolved binding sites that hitch them to this abundant and phylogenetically stable component of the cell.

\section{Evolution of E. coli chromosome}

\section{from Millicent Masters}

IT has been possible to observe the intact chromosome of about half-adozen species of bacteria. In each species the DNA was found to be a closed circle. The circular chromosome of the best studied species of bacterium, Escherichia coli, contains enough DNA to code for about 3,000 average-sized polypeptide chains. Specific functions have been assigned to about 500 of these genes and the well developed genetics of $E$. coli has permitted their positions on the chromosome to be determined. Genes specifying proteins with related functions such as isoenzymes catalysing the same reaction or groups of enzymes involved in sequential metabolic conversions may be either close together or distant on the circular chromosome. Some related genes are close together because they are transcribed together (that is, they form an operon). Other related genes however are close together without sharing such a common control mechanism. Still other related genes, such as the five genes concerned with pyrimidine synthesis or seven of the 11 genes responsible for arginine biosyn- 